\title{
Laser-induced Fluorescence Velocimetry of Xe II in the 30-cm NSTAR-type Ion Engine Plume
}

\author{
Timothy B. Smith*, Daniel A. Herman ${ }^{\dagger}$ and Alec D. Gallimore ${ }^{\ddagger}$ \\ Plasmadynamics and Electric Propulsion Laboratory, University of Michigan, Ann Arbor, MI 48105 \\ George J. Williams Jr. ${ }^{\S}$ \\ NASA Glenn Research Center, Cleveland, OH 44135
}

\begin{abstract}
We present laser-induced fluorescence (LIF) measurements of Xe II $\left({ }^{4} D_{7 / 2}\right)$ in the FMT2 ion engine plume. Three $605.1 \mathrm{~nm}$ beams from a ring dye laser are focused through a single lens into a submillimeter interrogation volume, from which we collect LIF at 529.2 nm. Fourier-transform deconvolution of the LIF spectra returns ion velocity distribution estimates along the three laser beam directions. From this, we calculate axial, radial and vertical components of the mean ion velocity and temperature from $1 \mathrm{~mm}$ to 300 $\mathrm{mm}$ downstream of the accelerator grid. The error propagation endemic to this off-axis multiplex LIF technique obscures the location of the neutralization plane in axial profiles of axial velocity, while radial sweeps of axial and vertical velocity show no discernable trend. Radial profiles of radial velocity, however, show increasing divergence with radial position.
\end{abstract}

\section{Introduction}

Laser-induced fluorescence (LIF) has gained popularity as a non-intrusive plume diagnostic in the last decade. Early use of LIF for EP concentrated on relatively high-density systems. Zimmerman and Miles ${ }^{1}$ developed a technique for measuring hypersonic wind-tunnel velocities via helium Doppler-shifted LIF in 1980. This technique was adapted for use in hydrazine and hydrogen arcjets in the early 1990s by Erwin ${ }^{2}$ and Liebeskind, ${ }^{3-8}$ both of whom used hydrogen Balmer- $\alpha$ line LIF to measure radial profiles of axial velocity. Ruyten and Keefer ${ }^{9}$ developed a multiplex LIF method to simultaneously measure axial and radial velocity components of an argon arcjet, using an optogalvanic cell as a stationary reference plasma.

LIF methods were quickly applied to lower-density EP systems, such as Hall thrusters and ion engines. Gaeta et al. pioneered the use of LIF as an erosion rate diagnostic in 1992. ${ }^{10,11}$ In 1994, Manzella ${ }^{12}$ reported the first use of a diode laser to excite the $5 d^{2} F_{7 / 2} \rightarrow 6 d^{2} D_{5 / 2}^{0}$ transition of singly-ionized xenon (Xe II) at $834.7 \mathrm{~nm}$, measuring axial and azimuthal velocity components in a Hall thruster plume. Subsequent Xe II LIF studies of electrostatic thrusters have concentrated on Hall thrusters, using either Manzella's $834.7 \mathrm{~nm}$ transition ${ }^{13-18}$ or the $5 d^{4} D_{7 / 2} \rightarrow 6 p^{4} P_{5 / 2}^{0}$ transition at $605.1 \mathrm{~nm} .{ }^{19-28}$

We present data obtained via $605.1 \mathrm{~nm}$ LIF in two off-axis multiplex experiments downstream of the FMT-2 accelerator grid at three thruster operating conditions. All experiments were performed in the large vacuum test facility (LVTF) at the University of Michigan's Plasmadynamics and Electric Propulsion Laboratory (PEPL). Radial sweeps at $1 \mathrm{~mm}$ and $50 \mathrm{~mm}$ downstream of the screen grid provided data at $12.7 \mathrm{~mm}$ intervals from the thruster centerline, until the signal degraded at $y=11.4 \mathrm{~cm}$ (for TH19) to $y=12.7 \mathrm{~cm}$ (for TH15). Axial sweeps on the centerline provided data from $1 \mathrm{~mm}$ to $30 \mathrm{~cm}$ downstream of the accelerator grid. This study is, to the best of our knowledge, the first set of Xe II LIF velocimetry measurements made in an ion thruster plume.

\footnotetext{
*Research fellow, PEPL. Senior Member AIAA. timsmith@umich.edu

${ }^{\dagger}$ Graduate student research assistant, PEPL. Student Member AIAA.

${ }^{\ddagger}$ Professor and Director, PEPL. Associate Fellow AIAA

$\S$ Senior research associate, Ohio Aerospace Institute. Member AIAA.

Copyright (c) 2004 by the American Institute of Aeronautics and Astronautics, Inc. The U.S. Government has a royalty-free license to exercise all rights under the copyright claimed herein for Governmental purposes. All other rights are reserved by the copyright owner.
} 


\section{Theory}

In LIF, the wavelength of a narrow-linewidth tunable laser is swept through an absorption line of a plasma species, such as singly-ionized xenon (Xe II). Focusing optics direct this laser beam along a "beamwise" direction vector $\hat{\mathbf{k}} \equiv \mathbf{k} /|\mathbf{k}|$, where $\mathbf{k}$ is the beam's wave vector. Collection optics on another (usually perpendicular) axis sample the fluorescence emitted by absorbing particles in the interrogation volume. This interrogation volume, an ellipsoid defined by the intersecting beams, can be less than a millimeter on a side. Wavelength filtering and lock-in detection keep the LIF signal from being swamped by the bright light background typical of EP devices.

\section{A. Line broadening}

Consider a light source with vacuum wavelength $\lambda$ and frequency $\nu=c / \lambda$. An observer who is stationary with respect to the light source will see light at the same frequency $\nu$. An observer moving towards the light source will see a bluer (i.e., higher-frequency) light than the stationary viewer, while an observer moving away from the light source will see a redder (i.e., lower-frequency) light.

In LIF, this Doppler effect appears as a shift in the resonant frequency $\nu_{0}$ as the laser is scanned over a very short frequency range. The change in photon frequency $\Delta \nu=\nu-\nu_{0}$ for a particle with velocity $\mathbf{v}$ passing through a light beam of wave vector $\mathbf{k}$ is

$$
\triangle \nu=-\frac{\mathbf{k} \cdot \mathbf{v}}{2 \pi} .
$$

Given a beamwise velocity component $v_{k} \equiv \mathbf{v} \cdot \hat{\mathbf{k}}$ where $\hat{\mathbf{k}} \equiv \mathbf{k} /|\mathbf{k}|$, Eqn. 1 gives the beamwise velocity-tofrequency transformations

$$
\begin{aligned}
\nu & =\left(1-\frac{v_{k}}{c}\right) \nu_{0} \\
v_{k} & =\left(1-\frac{\nu}{\nu_{0}}\right) c .
\end{aligned}
$$

A swarm of particles with a normalized velocity distribution $f(\mathbf{v})$ will also "see" the frequency of incoming photons shifted by the relative velocity of the particle in the direction of the photon. The resulting Doppler

lineshape will be shifted by the beamwise bulk velocity $u_{k}=\langle\mathbf{v} \cdot \hat{\mathbf{k}}\rangle$ and broadened by the thermal width of the beamwise distribution $f\left(v_{k}\right)$. The generalized Doppler lineshape $d(\nu)$, when properly normalized so that

$$
\int_{-\infty}^{\infty} d(\nu) d \nu=1
$$

is given by

$$
d(\nu)=\frac{c}{\nu_{0}} f\left(\left[1-\frac{\nu}{\nu_{0}}\right] c\right) .
$$

When $f\left(v_{k}\right)$ is a one-dimensional stationary Maxwellian of the form

$$
f_{m}\left(v_{k}\right)=\left(\frac{M}{2 \pi k T}\right)^{1 / 2} \exp \left(-\frac{M v_{k}^{2}}{2 k T}\right)
$$

Eqn. 5 takes the familiar form ${ }^{40}$

$$
d_{m}(\nu)=\frac{c}{\nu_{0}}\left(\frac{M}{2 \pi k T}\right)^{1 / 2} \exp \left(-\frac{M c^{2}}{2 k T}\left[\frac{\nu-\nu_{0}}{\nu_{0}}\right]^{2}\right) .
$$

In species with no hyperfine structure (hfs), the LIF spectrum $i(\nu)$ is the convolution of the Doppler broadening with the natural (or lifetime) broadening function

$$
l(\nu)=\frac{\triangle \nu_{n}}{2 \pi} \frac{1}{\left(\nu-\nu_{0}\right)^{2}+\left(\triangle \nu_{n} / 2\right)^{2}}
$$


where $\triangle \nu_{n}=A_{j} /(2 \pi)$ is the natural linewidth for state $j$. This Lorentzian function is usually much narrower for warm gases than the Doppler broadening. In this case, the natural broadening can be replaced by a Dirac delta function,

$$
i(\nu)=d(\nu) \otimes l(\nu) \approx d(\nu) \otimes \delta(\nu)=d(\nu),
$$

letting us directly transform the LIF spectrum into an excellent approximation of the beamwise velocity distribution. The similarity between LIF spectra and $f\left(v_{k}\right)$ is good enough that LIF measurements in barium $^{29-31}$ and $\operatorname{argon}^{32}$ plasmas have been reported as velocity distributions.

\section{B. Hyperfine structure}

In xenon (and other species with hfs), the LIF spectrum can be modeled as the convolution of the hyperfine splitting function $h(\nu)$ with natural and Doppler broadening functions,

$$
i(\nu)=h(\nu) \otimes l(\nu) \otimes d(\nu) .
$$

The hyperfine splitting $h(\nu)$ is a series of $n$ Dirac delta functions,

$$
h(\nu)=\sum_{j=1}^{n} p_{j} \delta\left(\nu-\nu_{j}\right)
$$

where $\nu_{j}$ is the $j^{\text {th }}$ hyperfine line center and $p_{j}$ is the $j^{\text {th }}$ hyperfine line intensity. The $5 d^{4} D_{7 / 2} \rightarrow 6 p^{4} P_{5 / 2}^{0}$ transition of Xe II has $n=19$ such hyperfine splittings.

Hyperfine structure consists of two components. Isotopic splitting is caused by the mass and nuclear volume differences between isotopes in the sample. Nuclear-spin splitting is caused by the coupling between the nuclear spin $\mathbf{I}$ and the total electronic angular momentum $\mathbf{J}$.

There are nine stable isotopes of xenon, seven of which have natural abundances greater than one percent. Each of these isotopes causes a slightly different transition energy $E_{i j}$, resulting in isotopic splitting. This model uses naturally-occurring xenon isotopic abundances ${ }^{33}$ and shifts for the $5 d^{4} D_{7 / 2}-6 p^{4} P_{5 / 2}^{0}$ transition taken from the fast ion-beam LIF surveys of Bingham et al. ${ }^{34}$ and Borghs et al. ${ }^{35}$

Seven of the nine stable isotopes of xenon have even atomic mass, resulting in no nuclear spin $\mathbf{I}$. The two isotopes with an odd atomic mass, however, have non-zero nuclear spin quantum numbers $I$. The lighter isotope, ${ }^{129} \mathrm{Xe}$, has $I=1 / 2$, while ${ }^{131} \mathrm{Xe}$ has $I=3 / 2$. These non-zero nuclear spins cause nuclear-spin splitting of the atomic energy levels. This nuclear-spin splitting is 1 to 2 orders of magnitude broader than the isotopic splitting, and provides most of the $5 d^{4} D_{7 / 2}-6 p{ }^{4} P_{5 / 2}^{0}$ line's characteristic shape.

The total angular momentum $\mathbf{F}=\mathbf{I}+\mathbf{J}$ takes quantum number values

$$
F=I+J, I+J-1, \ldots,|I-J|
$$

where $J$ is the total electronic angular momentum quantum number. ${ }^{36}$ The extra term energy due to nuclear-spin splitting is given by ${ }^{37}$

$$
E_{\mathrm{nss}}=A \frac{C}{2}+B D
$$

where $A$ is the nuclear magnetic dipole interaction constant, $B$ is the nuclear electric quadrupole interaction constant, and the terms

$$
C=F(F+1)-I(I+1)-J(J+1)
$$

and

$$
D=\frac{(3 C / 4)(C+1)-I(I+1) J(J+1)}{2 I(2 I-1) J(2 J-1)}
$$

contain the nuclear spin-orbit interactions. This model uses Bröstrom's nuclear-spin structure constants for the Xe II $5 d{ }^{4} D_{7 / 2}$ and $6 p{ }^{4} P_{5 / 2}^{0}$ energy levels. ${ }^{38}$

The transition rule for nuclear-spin splitting is $\triangle F \equiv F-F^{\prime}=[0, \pm 1]$, where $F$ is the upper and $F^{\prime}$ is the lower state's total angular momentum quantum number. (The zero-zero transition is forbidden, $F=0 \nrightarrow F^{\prime}=0$.) Figure 1 shows the fine structure and nuclear-spin splitting for the $5 d^{4} D_{7 / 2}-6 p{ }^{4} P_{5 / 2}^{0}$ line. 


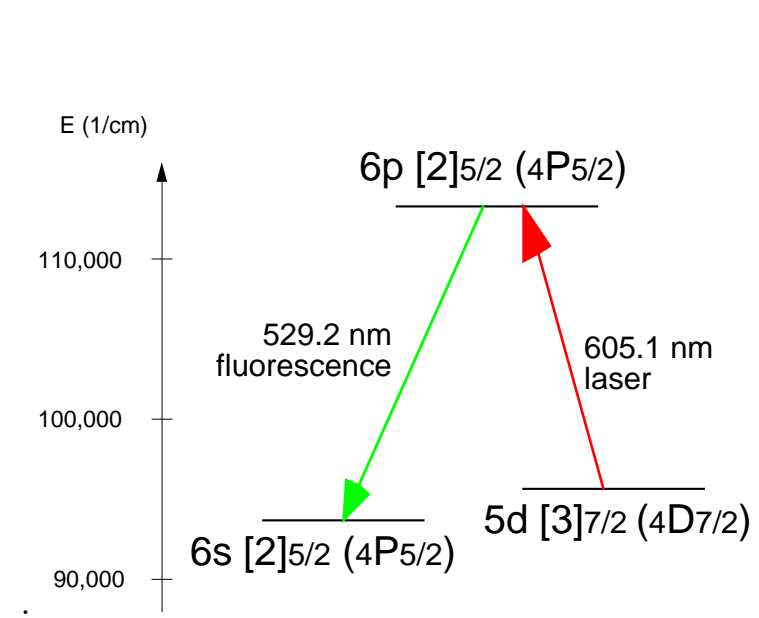

(a) Xe II LIF fine structure.

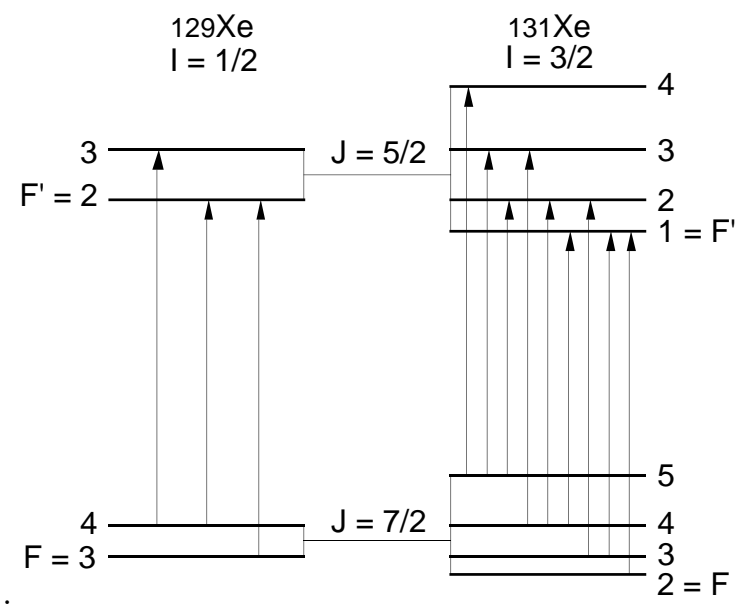

(b) Nuclear-spin structure of $605.1 \mathrm{~nm}$ line.

Figure 1. Fine and hyperfine structure of Xe II LIF.

The relative intensity of each nuclear-spin split component is given for a $J \rightarrow J-1$ transition by ${ }^{39}$

$$
\begin{aligned}
I(F \rightarrow F-1) & \propto \frac{P(F) P(F-1)}{F} \\
I(F \rightarrow F) & \propto \frac{(2 F+1)}{F(F+1)} P(F) Q(F) \\
I(F-1 \rightarrow F) & \propto \frac{Q(F) Q(F-1)}{F}
\end{aligned}
$$

where $P(F)=(F+J)(F+J+1)-I(I+1)$ and $Q(F)=I(I+1)-(F-J)(F-J+1)$.

For the isotopes with even mass numbers (i.e., without nuclear-spin splitting), the line intensity $p_{j}$ is linearly proportional to the naturally-occurring abundance for each isotope. The line intensities of ${ }^{129} \mathrm{Xe}$ and ${ }^{131} \mathrm{Xe}$ are linearly proportional to the product of the isotopic abundance and the relative intensity of the nuclear-spin split components. Table 1 presents hyperfine line intensities $p_{j}$ and line centers $\nu_{j}$ (relative to $\nu_{0}$, the line center for ${ }^{132} \mathrm{Xe}$ ) for the $605.1 \mathrm{~nm}$ absorption hyperfine splitting model of Eqn. 11.

\section{Deconvolution}

Figure 2(a) shows the LIF spectrum from a perfectly cold stationary plasma, where the velocity distribution $f(\mathbf{v})=\delta(\mathbf{v})$. This can be described by the convolution

$$
c(\nu)=h(\nu) \otimes l(\nu)
$$

so that Eqn. 10 becomes

$$
i(\nu)=c(\nu) \otimes d(\nu)
$$

Given the Fourier transforms $C(\tau)$ and $D(\tau)$ of the cold-plasma and Doppler broadening functions $c(\nu)$ and $d(\nu)$, the convolution theorem states that Eqn. 20 is equivalent to the product

$$
I(\tau)=C(\tau) D(\tau)
$$

where $I(\tau)$ is the Fourier transform of $i(/ n u)$. It then follows that the Fourier transform quotient

$$
D(\tau)=I(\tau) / C(\tau)
$$

is equivalent to the deconvolution

$$
d(\nu)=i(\nu) \oslash c(\nu)
$$


Table 1. Hyperfine line shifts and intensities for Xe II $5 d^{4} D_{7 / 2}-6 p^{4} P_{5 / 2}^{0}$.

\begin{tabular}{|c|c|c|c|c|c|c|c|c|c|c|c|}
\hline $\begin{array}{l}\text { line } \\
j\end{array}$ & $\begin{array}{c}\text { mass } \\
M \\
(\mathrm{amu}) \\
\end{array}$ & $\begin{array}{l}F \\
(-)\end{array}$ & $\begin{array}{l}F^{\prime} \\
(-)\end{array}$ & $\begin{array}{r}\text { shift } \\
\nu_{j}-\nu_{0} \\
(\mathrm{MHz})\end{array}$ & $\begin{array}{c}\text { intensity } \\
p_{j} \\
(-)\end{array}$ & $\begin{array}{l}\text { line } \\
j\end{array}$ & $\begin{array}{c}\text { mass } \\
M \\
(\mathrm{amu}) \\
\end{array}$ & $\begin{array}{l}F \\
(-)\end{array}$ & $\begin{array}{l}F^{\prime} \\
(-)\end{array}$ & $\begin{array}{r}\text { shift } \\
\nu_{j}-\nu_{0} \\
(\mathrm{MHz}) \\
\end{array}$ & $\begin{array}{c}\text { intensity } \\
p_{j} \\
(-) \\
\end{array}$ \\
\hline 0 & 124 & - & - & 336.6 & 0.000096 & 10 & 131 & 3 & 2 & -1335.2 & 0.037821 \\
\hline 1 & 126 & - & - & 252.4 & 0.000090 & 11 & 131 & 3 & 3 & 170.2 & 0.008273 \\
\hline 2 & 128 & - & - & 172.0 & 0.019200 & 12 & 131 & 3 & 4 & 2014.1 & 0.000236 \\
\hline 3 & 129 & 3 & 2 & 2718.3 & 0.110167 & 13 & 131 & 4 & 3 & -406.1 & 0.053186 \\
\hline 4 & 129 & 3 & 3 & -2194.8 & 0.005508 & 14 & 131 & 4 & 4 & 1437.8 & 0.006382 \\
\hline 5 & 129 & 4 & 3 & -182.5 & 0.14 & 15 & 131 & 5 & 4 & 641.3 & 0.072806 \\
\hline 6 & 130 & - & - & 83.6 & 0.040800 & 16 & 132 & - & - & 0.0 & 0.268900 \\
\hline 7 & 131 & 2 & 1 & -2000.4 & 0.026475 & 17 & 134 & - & - & -75.8 & 0.104400 \\
\hline 8 & 131 & 2 & 2 & -938.5 & 0.006304 & 18 & 136 & - & - & -140.9 & 0.088700 \\
\hline 9 & 131 & 2 & 3 & 566.9 & 0.000315 & & & & & & \\
\hline
\end{tabular}

which can be converted to a beamwise velocity distribution estimate by the transformation

$$
\hat{f}\left(v_{k}\right)=\frac{1}{\lambda_{0}} d\left(\left[1-\frac{v_{k}}{c}\right] \nu_{0}\right) .
$$

This simple inverse filter approach, though theoretically sound, quickly collapses for realistic signal-tonoise ratios. Deconvolution methods tend to preferentially amplify high-frequency noise. ${ }^{41}$ We have found ${ }^{28}$ that low-pass filtering of the deconvolved velocity distribution estimate $\hat{f}\left(v_{k}\right)$ with a Gaussian function reduces the noise amplification factor to an acceptable level, at the cost of discarding some high-frequency information along with the noise.

\section{Beam injection}

In the original multiplex technique developed by Keefer et al. ${ }^{9}$ a large focusing lens is placed so its optical axis is perpendicular to the thruster axis. Two parallel beams, which are chopped at different frequencies to aid phase-locked amplification, are directed to the lens. One beam, which passes through the center of the lens, is called the "radial" beam; the other, which enters the lens upstream of its center, is called the "axial"

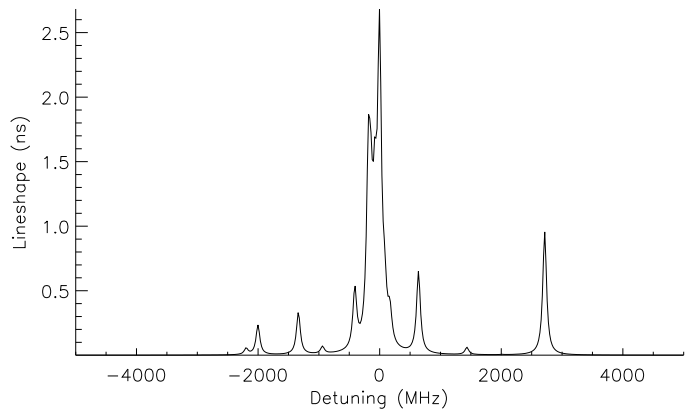

(a) Cold-plasma spectrum $c(\nu)$.

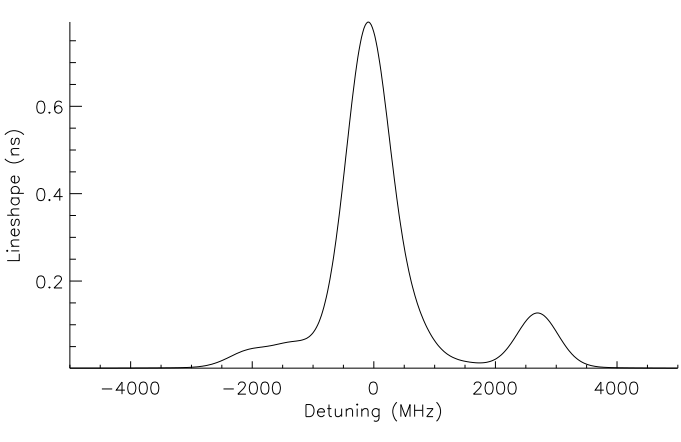

(b) Warm-plasma spectrum $w(\nu), T=600 \mathrm{~K}$.

Figure 2. Cold-plasma and warm-plasma spectra for the Xe II $5 d^{4} D_{7 / 2}-6 p^{4} P_{5 / 2}^{0}$ line 
beam. Both beams are focused by the lens, meeting at the LIF interrogation point. A collection lens, placed so its optical axis is perpendicular to both the thruster and focusing lens axes, sends LIF from both beams through a monochromator to a photomultiplier tube (PMT). The resulting current signal is passed to the lock-in amplifiers, which separate out each beam's LIF signal.

Fig. 3 shows the beam propagation axes relative to the thruster for the three-beam off-axis multiplex technique perfected by Williams et al. ${ }^{23-25}$ In this variant, the focusing lens axis is pointed downward, so that the center beam is "vertical" (rather than "radial"), with a direction vector $\hat{\mathbf{v}}$. The "offaxial" beam enters downstream of the lens center, emerging at an angle $\alpha$ from the vertical beam along a beam direction vector $\hat{\mathbf{a}}$, on a plane parallel to the thruster axis. Finally, the third (or "off-radial") beam enters to one side of the lens center, emerging at an angle $\beta$ from the vertical beam along a beam direction vector $\hat{\mathbf{r}}$, on a plane perpendicular to the thruster axis.

Decomposed onto a set of axes orthogonal to the thruster, the beam direction vectors are

$$
\begin{aligned}
\hat{\mathbf{v}} & =-\hat{\mathbf{z}} \\
\hat{\mathbf{a}} & =-\sin \alpha \hat{\mathbf{x}}-\cos \alpha \hat{\mathbf{z}} \\
\hat{\mathbf{r}} & =-\sin \beta \hat{\mathbf{y}}-\cos \beta \hat{\mathbf{z}} .
\end{aligned}
$$

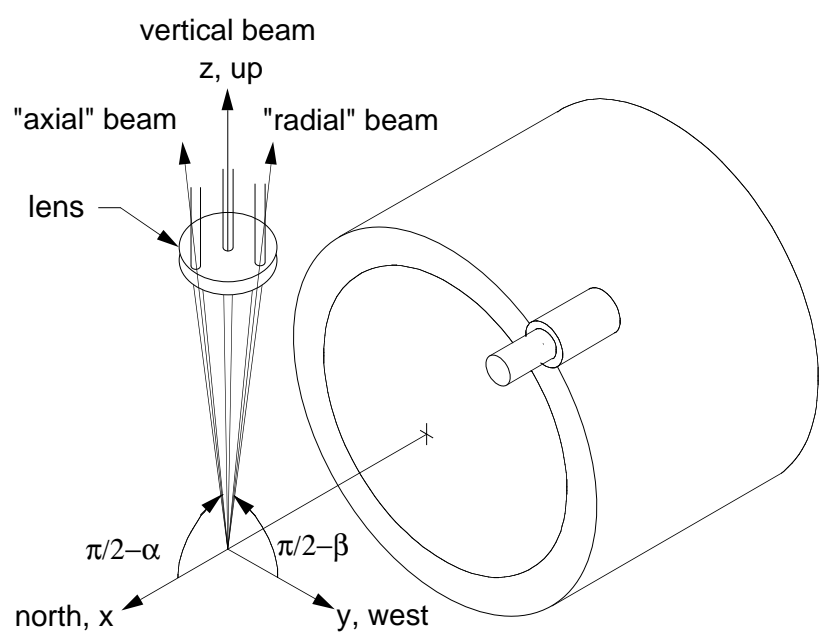

Thus, the beamwise bulk velocity components $u_{v}$, $u_{a}$ and $u_{r}$ (measured by the Doppler shift relative to a stationary reference plasma) can be readily transformed to thruster coodinates by

$$
\begin{aligned}
& u_{x}=-\frac{u_{a}+u_{v} \cos \alpha}{\sin \alpha} \\
& u_{y}=-\frac{u_{r}+u_{v} \cos \beta}{\sin \beta} \\
& u_{z}=-u_{v}
\end{aligned}
$$

Unfortunately, the small laser beam convergence angles needed to avoid vignetting can cause significant errors in the above transformation. Consider an off-axial velocity uncertainty of $\triangle u_{a}$; even in the absence of angular uncertainty or vertical velocity uncertainty, the true axial ( $x$-component) uncertainty is $\Delta u_{x}=\Delta u_{a} / \sin \alpha$. Furthermore, the proportional axial velocity uncertainty with respect to angular error is

$$
\frac{1}{u_{x}} \frac{\partial u_{x}}{\partial \alpha}=\frac{u_{z}}{u_{x}}-\cot \alpha
$$

Thus, both velocity errors and angular errors diverge rapidly at small angles. For instance, at $\alpha=10^{\circ}$, independant $2 \%$ random errors in $u_{z}$ and $u_{a}$, combined with a $2 \%$ bias in angular measurement, result in a $20 \%$ error in the calculated axial velocity $u_{x}$.

Transforming the beamwise temperatures to axes orthogonal to the thruster is less straightforward, as it requires two major assumptions. The first assumption, that the orthogonal velocity distribution projections

$$
\begin{aligned}
& f_{x}\left(v_{x}\right)=\int_{-\infty}^{\infty} \int_{-\infty}^{\infty} f(\mathbf{v}) d v_{y} d v_{z} \\
& f_{y}\left(v_{y}\right)=\int_{-\infty}^{\infty} \int_{-\infty}^{\infty} f(\mathbf{v}) d v_{x} d v_{z} \\
& f_{z}\left(v_{z}\right)=\int_{-\infty}^{\infty} \int_{-\infty}^{\infty} f(\mathbf{v}) d v_{y} d v_{z}
\end{aligned}
$$

are drifting Maxwellians, is implicit in the term "temperature," and is a reasonable way to quickly summarize the distribution in terms of bulk velocity and temperature components. The second assumption, that the velocity distributions along the orthogonal axes are statistically independant (i.e., $f(\mathbf{v})=f_{x}\left(v_{x}\right) f_{y}\left(v_{y}\right) f_{z}\left(v_{z}\right)$ ), 
is less supportable; any tilting of the two-dimensional velocity distribution contours with respect to the thruster axes makes this second assumption invalid. However, we currently have no reason to suppose that this assumption is not valid in electrostatic thruster plumes.

If we make this simplifying assumption, we can model the two-dimensional contours of $f_{x z}\left(v_{x}, v_{z}\right)$ and $f_{x y}\left(v_{x}, v_{y}\right)$ as untilted ellipses in velocity space. Since the velocity FWHM of a Maxwellian is

$$
\mathrm{FWHM}=\sqrt{8 \ln 2 \frac{k T}{M}}
$$

this untilted ellipse model implies that the off-axial temperature $T_{a}$, the axial temperature $T_{x}$ and the vertical temperature $T_{v}$ are related by

$$
\cos ^{2} \alpha+\left(\frac{T_{v}}{T_{x}}\right)^{2} \sin ^{2} \alpha=\left(\frac{T_{v}}{T_{a}}\right)^{2}
$$

Solving for the axial temperature yields

$$
T_{x}=T_{v}\left[\frac{\left(T_{v} / T_{a}\right)^{2}-1}{\cos ^{2} \alpha}+1\right]^{-1 / 2}
$$

for $\alpha \neq 0$ and $T_{v} / T_{a}>\sin \alpha$. By the same train of logic, the radial (i.e., $y$-component) temperature is

$$
T_{y}=T_{v}\left[\frac{\left(T_{v} / T_{r}\right)^{2}-1}{\cos ^{2} \beta}+1\right]^{-1 / 2}
$$

for $\beta \neq 0$ and $T_{v} / T_{r}>\sin \beta$.

\section{Apparatus and procedure}

\section{A. Thruster}

Figure 4 shows the FMT-2 ion thruster, one of the two 2.3-kW functional model thrusters (FMTs) developed as immediate predecessors to the engineering model (EMT) NSTAR thrusters. The EMT thrusters are the principal ground test versions of the NASA Solar Electric Propulsion Technology Application Readiness (NSTAR) $\phi 30 \mathrm{~cm}$ ion engine, which was successfully used as the primary propulsion for the Deep Space 1 (DS-1) probe. Unlike the EMT, the FMT makes extensive use of 1100 grade (i.e., soft) aluminum for components with low thermal loads or erosion rates. The discharge cathode and ion optics are identical to those used in the EMTs and flight thrusters (FTs).

The FMT-2 was assembled and modified at NASA GRC specifically for use at PEPL. These modifications include the addition of windows to the discharge chamber wall and plasma screen, allowing optical access for internal LIF studies. ${ }^{25}$ Three $102 \mathrm{~mm}$ x $32 \mathrm{~mm}$ x $3 \mathrm{~mm}$ quartz windows are mounted in the top, bottom and right-hand side (looking downstream) of the discharge chamber wall, with the discharge cathode exit plane passing roughly just upstream of the window centers. Though the EMT and

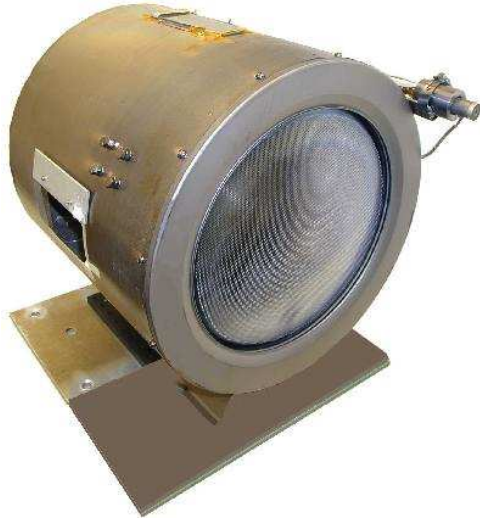

Figure 4. Photograph of the FMT-2 ion thruster.

FT plasma screens are conformal, the FMT plasma screen is cylindrical, facilitating window placement. Two $127 \mathrm{~mm} \times 45 \mathrm{~mm} \times 1.5 \mathrm{~mm}$ quartz windows are mounted in the top and bottom of the plasma screen, while a $127 \mathrm{~mm} \times 76 \mathrm{~mm} \times 1.5 \mathrm{~mm}$ window on the plasma screen side reduces vignetting of the LIF signal.

The discharge and overall engine performance of the FMT at PEPL has been nearly identical to that of the flight engine over the entire throttling range of the NSTAR thruster.

\section{B. Facility}

All tests were performed in the $\phi 6 \times 9 \mathrm{~m} \mathrm{LVTF}$ at PEPL. An adjustable $1.8 \times 2.2 \mathrm{~m}$ graphite beam dump protected the north end cap and suppressed back sputtering caused by the ion beam. Four of the seven 
available CVI Model TM-1200 Re-Entrant Cryopumps, each surrounded by a liquid nitrogen baffle, provided a xenon pumping speed measured at $140,000 \mathrm{l} / \mathrm{s}$ with a base pressure of less than $2 \times 10^{-7}$ Torr. Two hotcathode gauges, a Varian model 571 on a valved extension to the LVTF wall and a Varian model UHV-24 nude gauge mounted inside the chamber, monitored the chamber pressure at high vacuum. Propellant flow to the discharge cathode, discharge chamber and neutralizer was manually controlled by needle valves and monitored by three Teledyne Hastings NALL-100G flowmeters, calibrated by a bubble flow meter to an accuracy within NASA specifications. ${ }^{25}$

The FMT was positioned on an axially-adjustable work platform spanning the LVTF centerline, which supports a custom positioning system developed by New England Affiliated Technologies (NEAT). This system consists of a $1.8 \mathrm{~m}(6 \mathrm{ft})$ linear stage in the radial (east-west) direction, mounted on a $0.9 \mathrm{~m}(3 \mathrm{ft}$ ) linear stage in the axial (north-south). Both lateral stages are PC-controlled by a custom LabView VI, with locational resolution on the order of $0.25 \mathrm{~mm}$.

\section{Laser and external optics}

The laser system used in these experiments is a Coherent 899-29 Autoscan ring dye laser. This PC-controlled system has a nominal linewidth of $500 \mathrm{kHz}$, tuning repeatability of $50 \mathrm{MHz}$ and a scanning range of over 100 $\mathrm{GHz}$ (in $10 \mathrm{GHz}$ segments). PC-controlled scanning and data collection are synchronized by the Autoscan software.

Pumping for this dye laser is provided by an Coherent SBRC-R-DBW20/4 argon-ion laser, with a nominal broadband power rating of $25 \mathrm{~W}$. With the intercavity assembly (ICA) removed, the dye laser can generate up to $2 \mathrm{~W}$ of tunable broadband light using Rhodamine- $6 \mathrm{G}$ dye at $605 \mathrm{~nm}$. With the ICA installed, the same system can provide anywhere from 300 to $450 \mathrm{~mW}$ of narrow-linewidth light at $605.1 \mathrm{~nm}$.

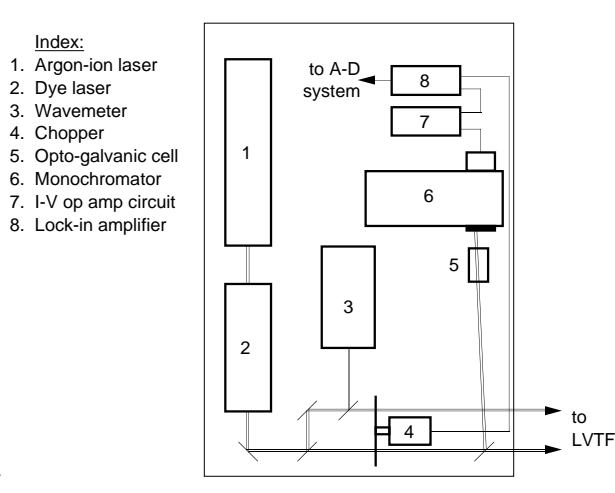

(a) Laser division and modulation.

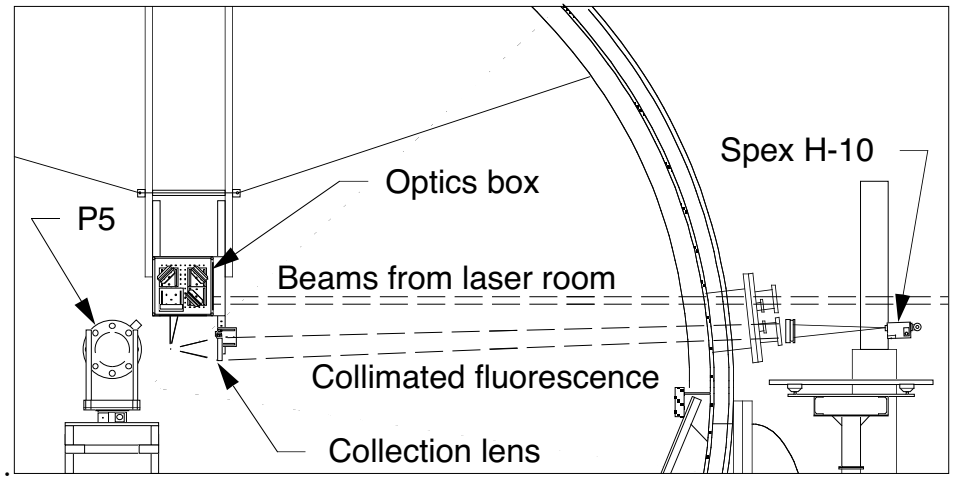

(b) Beam delivery and collection optics.

Figure 5. Optics and instrumentation schematic.

Fig. 5(a) shows a schematic of the optical table contents, which include the laser system, wavemeter, choppers and beamsplitting optics. A controlled atmosphere/low-dust enclosure (usually referred to as the laser room) protects these from the rest of the lab ${ }^{\mathrm{a}}$. A high-reflecting $\phi 25 \mathrm{~mm}$ mirror directs the laser beam into the conditioning optics. The first optic in this train is a $25 \mathrm{~mm}$ x $25 \mathrm{~mm}$ x $1.6 \mathrm{~mm}$ quartz slide, which sends a sampling beam to a Burleigh WA1000 wavemeter with a $0.1 \mathrm{pm}$ resolution and a $1.0 \mathrm{pm}$ accuracy between $400 \mathrm{~nm}$ and $1 \mu \mathrm{m}$.

For off-axis multiplex LIF, we split the laser output into three beams with two $25 \mathrm{~mm} \times 25 \mathrm{~mm} \times 1.6$ $\mathrm{mm}$ parallel-plate beamsplitters. Small high-reflecting mirrors on kinematic mounts send these beams down the LVTF beam tube, while micrometer stages holding the kinematic mounts allow fine adjustment of the distance between parallel beams. Since the large natural fluorescence at $529.2 \mathrm{~nm}$ would otherwise drown out the LIF signal, we chop the laser beam to permit phase-lock amplification of the LIF signal. Two beams pass through a Stanford SR541 two-frequency optical chopper, while the third beam passes through another

\footnotetext{
${ }^{\mathrm{a}}$ And vice versa.
} 
chopper. The frequencies of all three beams must be kept well away from harmonics of the other beams; otherwise, aliasing within the lock-in amplifiers can cause cross-talk between LIF signals.

Another $25 \mathrm{~mm}$ quartz slide downstream of the chopper sends a sampling beam through the center of a Hamamatsu L2783-42 XeNe-Mo hollow-cathode optogalvanic cell filled with a Xe-Ne gas mixture. ${ }^{25}$ A $250 \mathrm{~V}$ discharge across this optogalvanic cell gives a strong Xe II LIF signal, collected by a Chromex 500is monochromator with a Hamamatsu 928 photo-multiplier tube (PMT). An equivalent optogalvanic signal can be detected in the AC voltage drop across the cell's ballast resistor. Deconvolution of either signal provides a stationary reference for the distributions extracted from plume LIF.

Two $\phi 100 \mathrm{~mm}$ protected silver mirrors in a periscope configuration (with the upfold mirror on the optical table and the downfold mirror on the LVTF window waterline) send the primary beams out of the laser room, down the beam tube and into the LVTF. Absorbent material lining the beam tube walls reduces internal reflections, and helps absorb reflections from the LVTF windows.

Figure 5(b) shows the LVTF beam handling setup for the off-axis multiplex technique. The LVTF optics box is a $318 \mathrm{~mm} \times 394 \mathrm{~mm} \times 220 \mathrm{~mm}$ graphite-lidded enclosure with anti-reflection (AR) coated windows, which protect its contents from sputtering deposition and erosion. This enclosure contains three square $100 \mathrm{~mm}$ mirrors on kinematic mounts, used to direct all three incoming beams through a focusing lens. Small adjustments of the $\phi 100 \mathrm{~mm}$ upfold and downfold mirrors in the laser room steer the vertical beam to the center of the focusing lens. This lens focuses all three beams to sub-millimeter beam waists at the interrogation volume. During testing, this point remains fixed in space. To take LIF spectra at different points in the plume, we translate the thruster around the interrogation volume.

We use a small level during setup to ensure that the upper surface of this lens is level, and then temporarily place a second-surface mirror atop the focusing lens mount during setup and realignment. Small adjustments of the square $100 \mathrm{~mm}$ mirrors steer the retroreflected spots back to the laser room, ensuring that the vertical beam is plumb. When the retroreflected beam spots on the laser room upfold mirror overlay the original beam spots, the beams are also parallel. We measure beam spacing by replacing the retroreflection mirror with a gridded card, photographing the beam spots, and measuring the distances between spot centers in Photoshop; the standard deviation of multiple measurements provides an estimate of beam angle uncertainty.

The FMT has a $\phi 0.2 \mathrm{~mm}$ tungsten wire loop attached to the forward edge of the side plasma screen window to facilitate laser alignment. Two separate AR windows protect the $\phi 100 \mathrm{~mm}, f / 2.5$ collection lens. After placing the laser focal volume on the alignment feature, we adjust the collection lens, sending a collimated ${ }^{\mathrm{b}}$ beam of scattered light through the LVTF window. During experiments, the collected fluorescence follows the same path.

The collimated fluorescence from the thruster plume is focused by a $\phi 100 \mathrm{~mm}, f / 5$ lens onto a Spex H-10 monochromator with a Hamamatsu 928 PMT. This monochromator acts as a linewidth filter centered on the $529.2 \mathrm{~nm}$ fluorescence line. By holding a second-surface mirror flat against the monochromator entrance slits, we can use retroreflection of scattered light from the alignment pin to determine if the monochromator is aligned with the collection optics axis. Micrometer-driven rotation stages allow fine tilt and pan adjustment of the monochromator body. Stanford SR810 and SR850 DSP lock-in amplifiers, using a 1-second time constant, isolate the fluorescence components of these signals.

The Coherent 899-29 laser's Autoscan software collects and matches laser wavelength to the corresponding lock-in output. A scan rate of $60 \mathrm{~s} / 10 \mathrm{GHz}$ has proven to be sufficiently slow to ensure a reasonable signalto-noise ratio in most cases.

\section{Results}

We took three sets of data over two separate pumpdowns at thruster operating conditions TC 0 through TC 3. Both TC 0 and TC 1 were unneutralized, as the neutralizer failed to light for the first run. TC 0 and TC 2 replicated nominal full-power operating conditions for the FMT (1100 V screen potential and $1.75 \mathrm{~A}$ beam current), while TC 1 and TC 3 were run at atypically high beam current (1.78 A) and screen potentials. Our first attempt at high-power operation, TC 1, suffered from continual recycling, apparently caused by cyclic thermal expansion and sagging of the FMT's soft aluminum body. This made steady LIF measurement very difficult, and continued operation at TC 1 impractical. Subsequent high-voltage tests (TC 3$)$ were at a slightly lower screen potential $(1350 \mathrm{~V})$, retaining the 1.79 A beam current.

Radial sweeps at $1 \mathrm{~mm}$ and $50 \mathrm{~mm}$ downstream of the screen grid provided data at $12.7 \mathrm{~mm}$ intervals

\footnotetext{
${ }^{\mathrm{b}}$ Or slightly focusing, in order to avoid vignetting by the LVTF exit window.
} 
Table 2. FMT-2 operating conditions.

\begin{tabular}{lc|rrrr|c}
\hline \hline & & $\begin{array}{r}\text { TC 0 } \\
\text { (run 1) }\end{array}$ & $\begin{array}{r}\text { TC 1 } \\
\text { (run 1) }\end{array}$ & $\begin{array}{r}\text { TC 2 } \\
\text { (run 2) }\end{array}$ & $\begin{array}{r}\text { TC 3 } \\
\text { (run 2) }\end{array}$ & units \\
\hline Discharge voltage & $V_{b}$ & 25.56 & 25.00 & 25.38 & 24.86 & $\mathrm{~V}$ \\
Discharge current & $I_{d}$ & 12.88 & 13.08 & 12.60 & 12.16 & $\mathrm{~A}$ \\
Beam current & $I_{b}$ & 1.75 & 1.78 & 1.74 & 1.79 & $\mathrm{~A}$ \\
Accelerator potential & $V_{a}$ & -180.0 & -241.1 & -180.0 & -245.0 & $\mathrm{~V}$ \\
Screen potential & $V_{s}$ & 1100 & 1429 & 1102 & 1325 & $\mathrm{~V}$ \\
Main flow rate & $\dot{m}_{m}$ & 22.6 & 22.6 & 22.6 & 22.6 & sccm \\
Discharge cathode flow rate & $\dot{m}_{c}$ & 3.80 & 4.20 & 3.80 & 4.20 & sccm \\
Neutralizer cathode flow rate & $\dot{m}_{n}$ & 0.00 & 0.00 & 3.00 & 3.20 & sccm \\
Facility pressure & $P$ & 2.9 & 2.9 & 2.9 & 2.9 & $\mu$ Torr \\
\hline \hline
\end{tabular}

from the thruster centerline, until the signal degraded at between 11.4 and $12.7 \mathrm{~cm}$. Axial sweeps on the centerline provided data from $1 \mathrm{~mm}$ to $30 \mathrm{~cm}$ downstream of the accelerator grid.

\section{A. Radial sweeps near the exit plane}

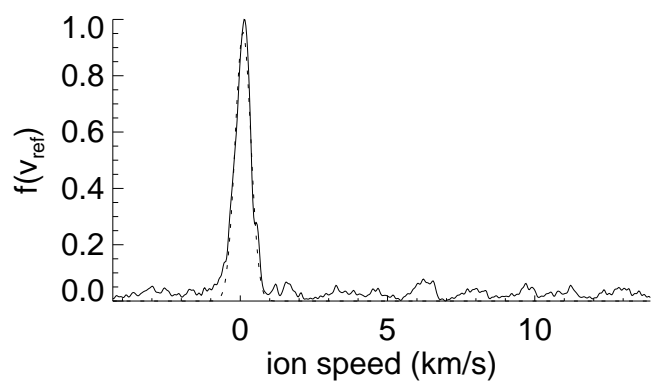

(a) Reference cell.

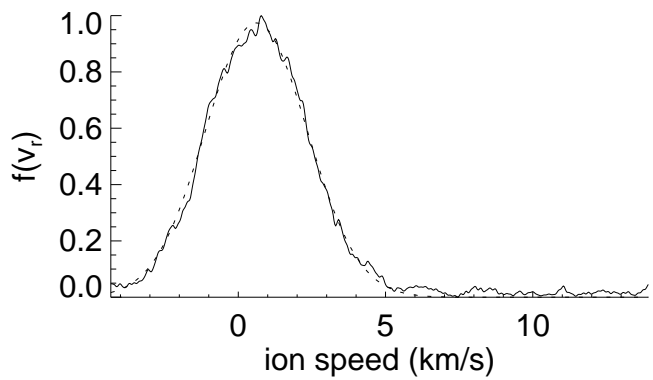

(c) "Radial" beam.

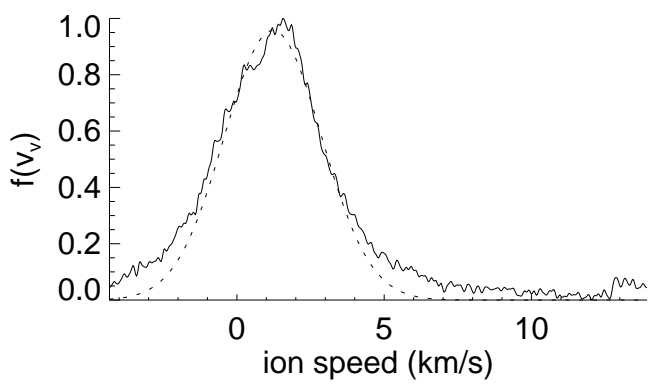

(b) Vertical beam.

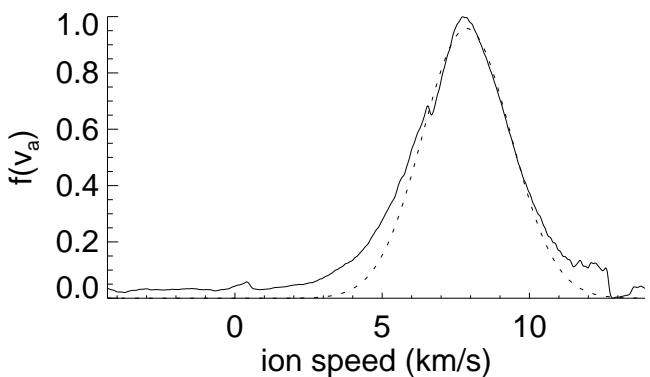

(d) "Axial" beam.

Figure 6. Deconvolved $\mathbf{f}(\mathbf{v})$ \& curve-fit at TC $\mathbf{0},(x, y)=(0.140,0.000) \mathbf{c m}$.

Figure 6 shows typical velocity distributions taken just downstream of the exit plane at TC 0 . The solid 
line is the deconvolved distribution, while the dashed line is a Maxwellian curve-fit to a user-defined area within the major peak. Some postprocessing of the original laser scans was needed to avoid "scan joints," computational artifacts caused by the dye laser's attempts to recover the end point of the previous $10-\mathrm{GHz}$ scan segment during an extended scan.
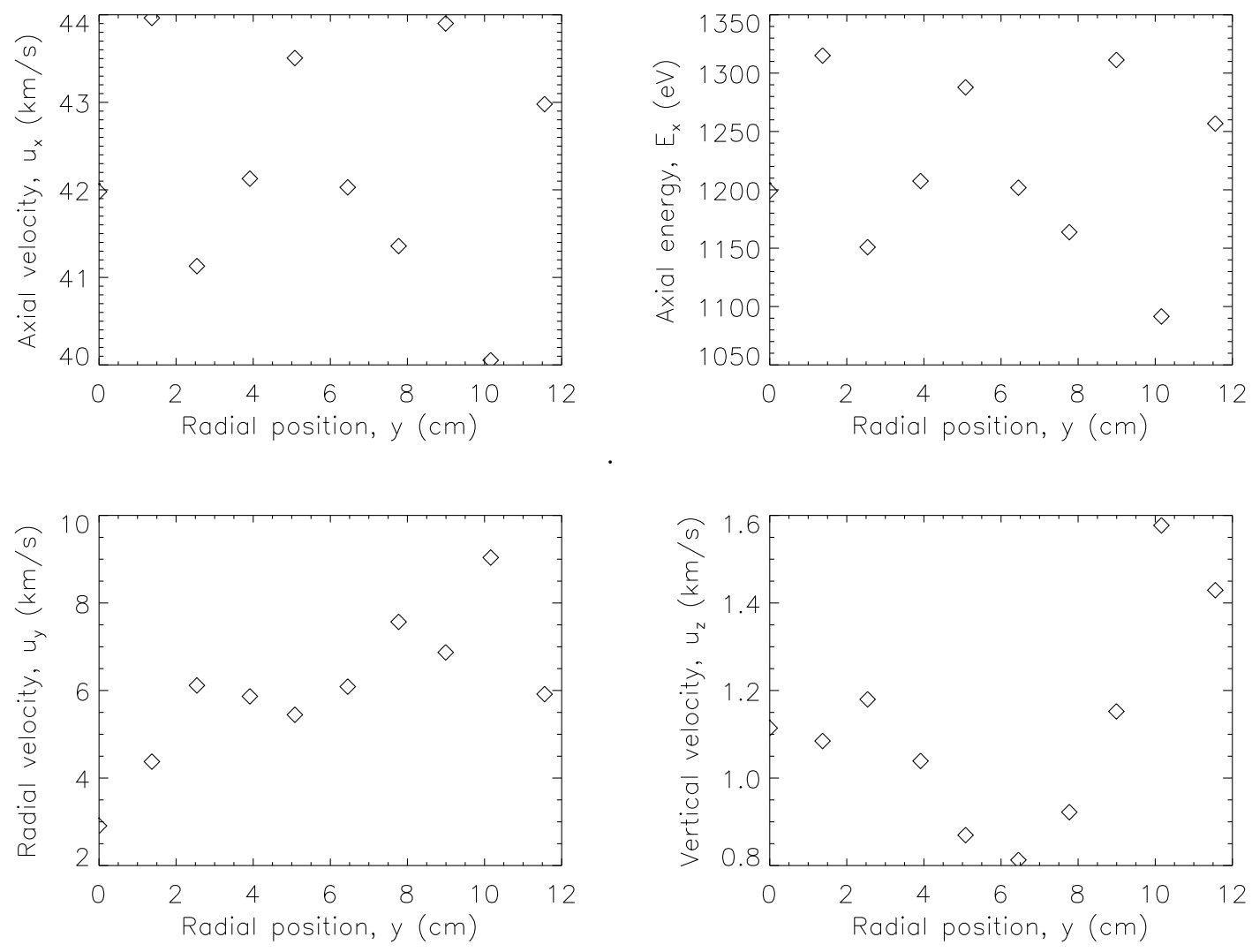

Figure 7. Ion velocity \& energy vs. radial position at $x=0.142 \mathrm{~cm}$ for TC 0 .

Figure 7 shows the radial variation of ion velocity and energy along a plane $1.40 \mathrm{~mm}$ downstream of the FMT-2 accelerator grid at TC 0 . The ion current immediately downstream of the accelerator grid is very highly concentrated into beamlets that pass through the grid apertures. Because of this concentrated ion current density, we had to hunt around the initial target radius in order to find a spot with enough ion density to provide a strong, clean LIF signal. This shows up in the somewhat irregular pattern of radial locations probed in this test.

Reasonable values for the ion axial energy $E_{x}$ lie between the screen potential $V_{s}$ and the total voltage $V_{T} \equiv V_{s}-V_{a}$; at TC0, $V_{s}=1100 \mathrm{~V}$ and $V_{T}=1280 \mathrm{~V}$. Three points lie outside this range, but the mean axial velocity $(42.3 \mathrm{~km} / \mathrm{s})$ remains reasonable, and the maximum velocity error is within $4 \%$ of the mean. Radial velocity tends to increase linearly with radial position, with a centerline radial velocity of $2.91 \mathrm{~km} / \mathrm{s}$ that suggests the thruster axis is pointed slightly $\left(3.9^{\circ}\right)$ eastward. The vertical velocity is uniformly positive, with a mean value $(1.12 \mathrm{~km} / \mathrm{s})$ that suggests the thruster is tilted slightly $\left(1.5^{\circ}\right)$ upwards.

Figure 8 shows the radial variation of ion velocity and energy $1.42 \mathrm{~mm}$ downstream of the FMT-2 accelerator grid at TH19. Only one point is outside the expected $V_{s} \leq E_{x} \leq V_{T}$ range; the mean axial velocity is $46.2 \mathrm{~km} / \mathrm{s}$, and the maximum velocity error is within $3.9 \%$ of the mean. Except for one point on the centerline, radial velocity tends to increase linearly with radial position, while vertical velocity shows no clear trend. 

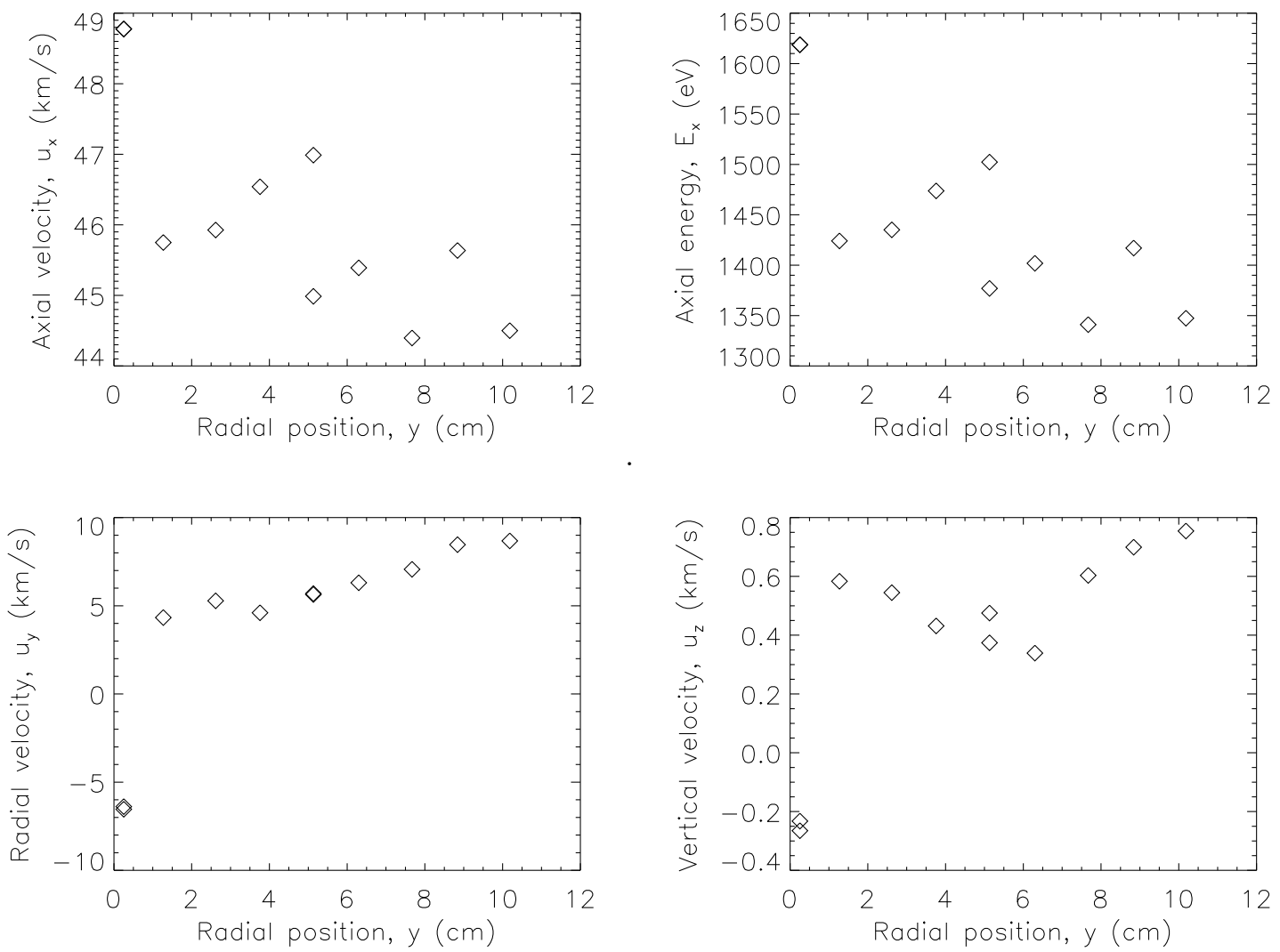

Figure 8. Ion velocity \& energy vs. radial position at $x=0.142 \mathrm{~cm}$ for TC 3.

\section{B. Radial sweeps, $5 \mathrm{~cm}$ downstream}

Figure 9 shows the radial variation of ion axial velocity and energy along a plane $5.090 \mathrm{~cm}$ downstream of the FMT-2 accelerator grid at TC 1 . Screen potential at this condition is $1429 \mathrm{~V}$, while the total voltage is 1670 $\mathrm{V}$. Ion axial energies for all but one point lie in the range $V_{s} \leq E_{x} \leq V_{T}$ at this condition, with a mean axial velocity of $47.5 \mathrm{~km} / \mathrm{s}$ and a maximum velocity error within $6.1 \%$ of the mean. Radial velocity again tends to increase linearly with radial position, with a centerline radial velocity that suggests the thruster axis is pointed still more sharply $\left(6.5^{\circ}\right)$ eastward and a maximum beam divergence of $3.3^{\circ}$ at the outermost radius. Likewise, the vertical velocity is again uniformly positive, with a mean value $(1.07 \mathrm{~km} / \mathrm{s})$ that suggests the thruster is tilted slightly $\left(1.3^{\circ}\right)$ upwards.

Figure 10 shows the radial variation of ion axial velocity and energy $5.10 \mathrm{~cm}$ downstream of the FMT-2 accelerator grid at TC 3. Screen potential at this condition is $1325 \mathrm{~V}$, while the total voltage is $1570 \mathrm{~V}$. Ion axial energies overlap both sides of the range $V_{s} \leq E_{x} \leq V_{T}$ at this condition. This anomalous ion energy spread can be explained by extremely noisy velocity distributions, making any bulk velocity predictions highly suspect for these data points.

\section{Axial sweeps}

Figure 11 shows the axial variation of ion axial velocity and energy along the FMT-2 centerline at TC 2. Screen potential at this condition is $1102 \mathrm{~V}$, while the total voltage is $1282 \mathrm{~V}$. Ion axial energies for all but one point lie in the range $V_{s} \leq E_{x} \leq V_{T}$ at this condition, with a mean axial velocity of $41.2 \mathrm{~km} / \mathrm{s}$ and a maximum velocity error within $5.0 \%$ of the mean. Both axial velocity and energy fall off with axial distance more-or-less linearly with $\ln (x)$.

Figure 12 shows the axial variation of ion radial and vertical velocity along the same centerline. Both radial and vertical velocities are uniformly positive. The mean centerline radial velocity $(3.66 \mathrm{~km} / \mathrm{s}) \mathrm{suggests}$ 

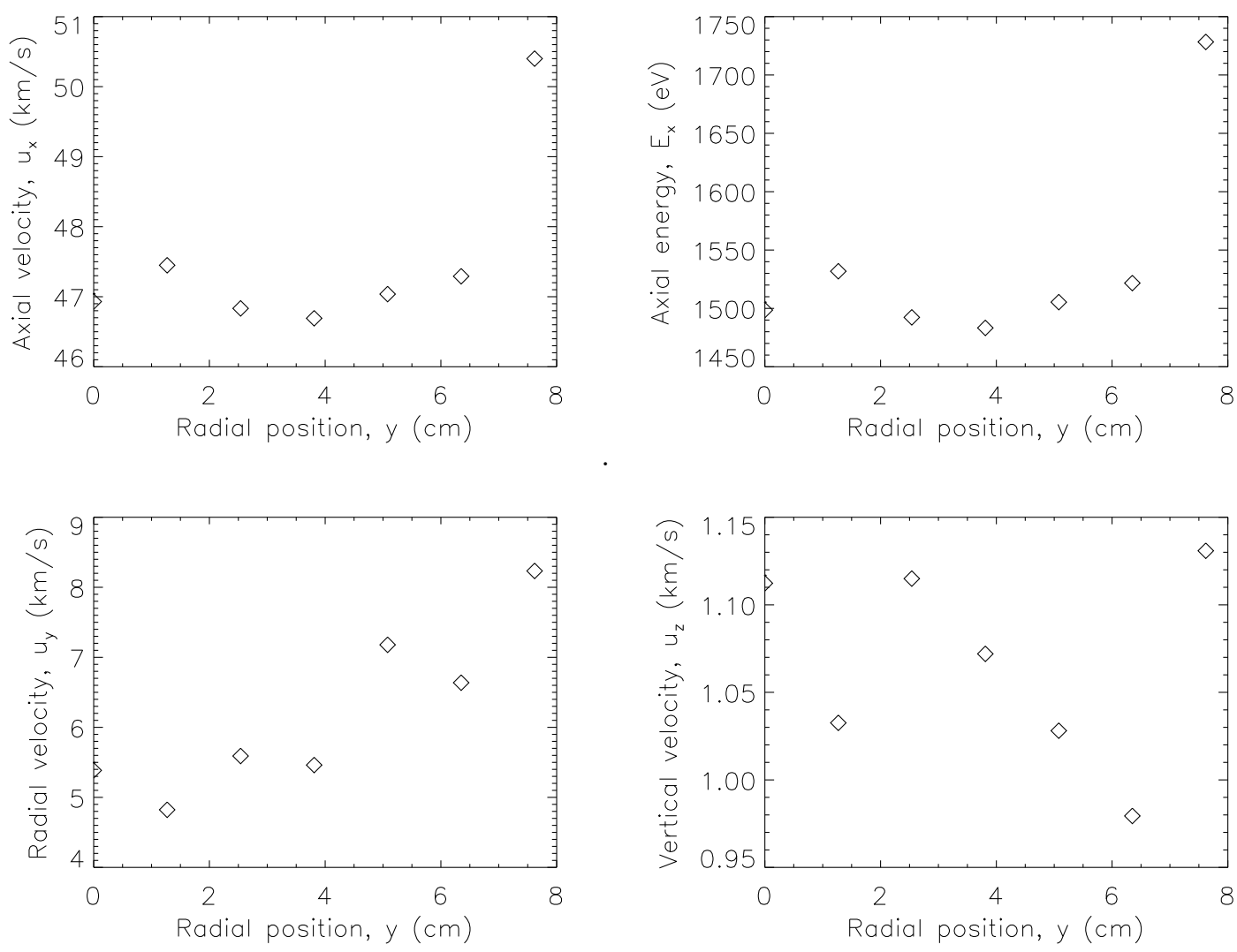

Figure 9. Ion velocity \& energy vs. radial position at $x=5.090 \mathrm{~cm}$ for TC 1.

a $5.1^{\circ}$ eastward misalignment of the thruster axis, while the mean vertical velocity $(0.31 \mathrm{~km} / \mathrm{s})$ suggests a mere $0.43^{\circ}$ upwards tilt.

\section{Conclusions}

This study is, to the best of our knowledge, the first set of Xe II LIF velocimetry measurements made downstream of an ion thruster. The highly-concentrated nature of the beamlets immediately downstream of the accelerator grid makes extreme near-field LIF at a regular series of radial positions difficult; getting a good overlap between the interrogation volume and a beamlet requires a bit of searching around the desired values. The LIF spectrums' signal-to-noise ratios (SNRs) quickly diminish with increasing radial distance, but the overall beam is so well collimated that the SNR is still quite good at the translation table's limit of axial travel.

Radial profiles of radial velocity showed a strongly increasing beam divergence with radial position. This is readily explained as an consequence of the grid design. Soulas ${ }^{42}$ points out that the apertures of the NSTAR screen and accelerator grids were designed to be aligned throughout the grids radius, with no beamlet steering to collimate the beam. As a result, beamlets are directed normal to the dished surface of the grids.

With the exception of a few anomalous points taken at unstable operating conditions, axial ion energies remained within a range between the screen potential potential $V_{s}$ and the total voltage $V_{T}$. Ideally, we should have seen ion energies dropping from $V_{T}$ to a value near $V_{s}$ at a clearly identifiable neutralization plane. Axial velocity trends, though, remain obscured by the large axial velocity uncertainty. Though we have previously shown that direct measurement of axial velocities by axial-injection LIF can provide velocity measurements with less than $2 \%$ precision error, ${ }^{27}$ the small interrogation angles required by the off-axis multiplex method drive the axial velocity error up to $20 \%$. As a result, we were unable to identify the 

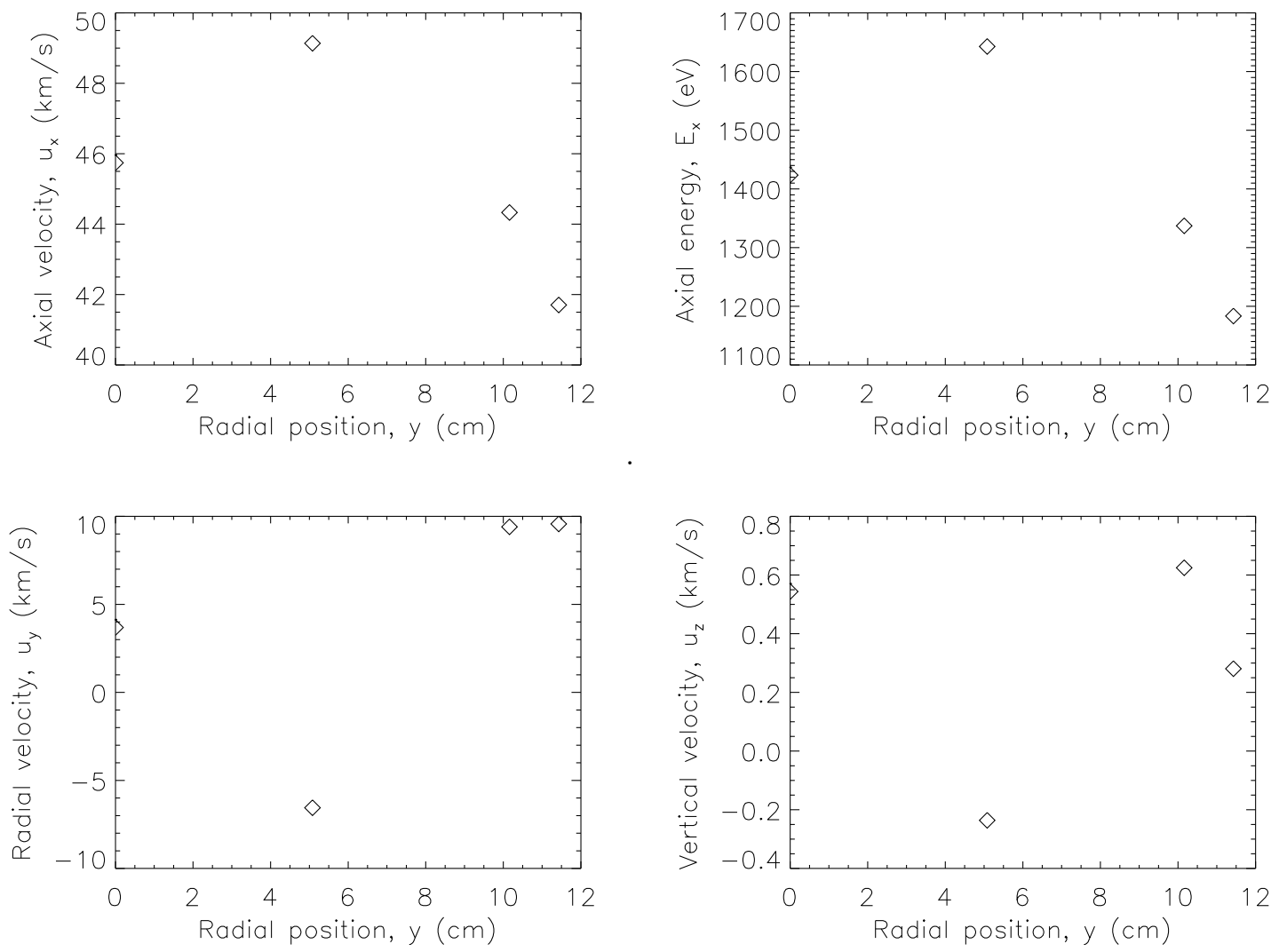

Figure 10. Ion velocity \& energy vs. radial position at $x=5.100 \mathrm{~cm}$ for TC 3 . 

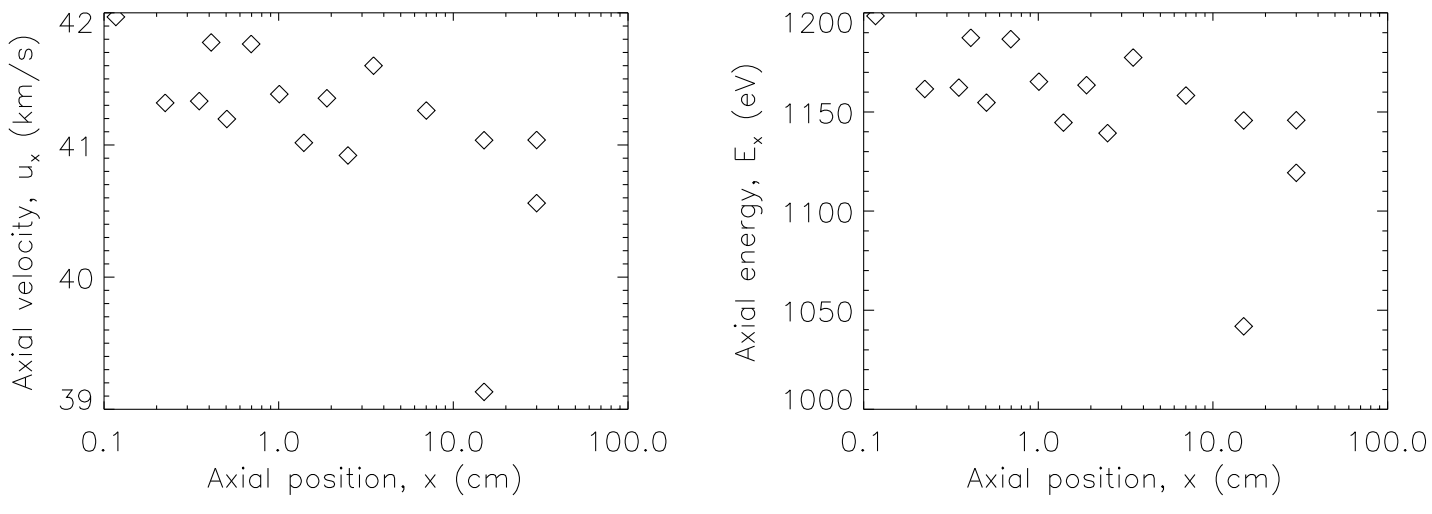

Figure 11. Axial ion velocity \& energy vs. axial position at $y=0.000 \mathrm{~cm}$ for TC 2 .

FMT-2 neutralization plane in this study. Future axial-injection LIF experiments in the FMT plume may have better success.

\section{Acknowledgements}

This research was supported by the Air Force Office of Scientific Research grants F49620-00-1-0201 and F49620-01-1-0061 (Dr. Mitat Birkan is the contract monitor for both). D.A.H. acknowledges support from the NASA Glenn Research Center via Grant NAG3-2216, monitored by J. Sovey and M. Domonkos. The authors are greatly appreciative of this support.

We would also like to thank the graduate and undergraduate students at PEPL for their assistance in experimental setup and operation, and the Department's technical support staff for their aid with facility repairs and modifications.

\section{References}

${ }^{1}$ M. Zimmerman and R. B. Miles. "Hypersonic-helium-flowfield measurements with the resonant Doppler velocimeter." Applied Physics Letters 37(1980), 885-887.

${ }^{2}$ D. A. Erwin, G. C. Pham-Van-Diep and W. D. Dieninger. "Laser-induced fluorescence measurements of flow velocity in high-power arcjet thruster plumes." AIA A Journal 29(1991), 1298-1303.

${ }^{3}$ J. G. Liebeskind, R. K. Hanson and M. K. Cappelli. "Velocity measurements in a hydrogen arcjet using LIF." In Proceedings of the 24th AIAA Plasmadynamics and Lasers Conference. AIAA-91-2112, June 1991.
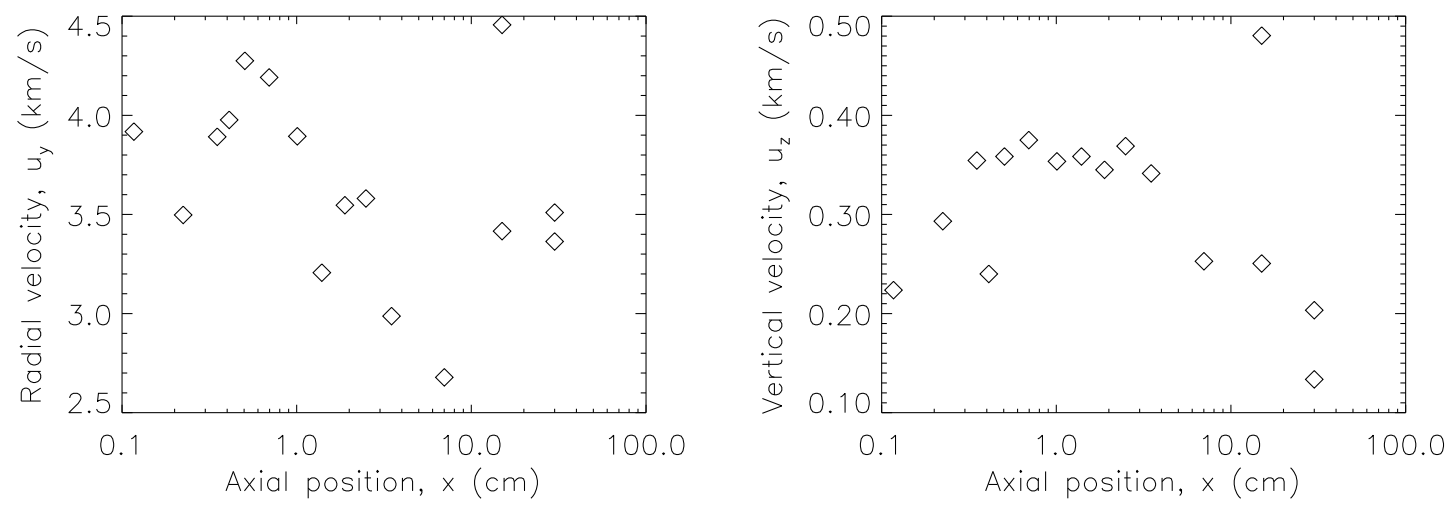

Figure 12. Radial \& vertical ion velocity vs. axial position at $y=0.000 \mathrm{~cm}$ for TC 2. 
${ }^{4}$ J. G. Liebeskind, D. H. Manzella, C. R. Ruthling, R. K. Hanson and M. K. Cappelli. "Optical diagnostics of a low power hydrogen arcjet." In Proceedings of the 22nd International Electric Propulsion Conference. IEPC-91-091, October 1991.

${ }^{5}$ J. G. Liebeskind, R. K. Hanson and M. K. Cappelli. "LIF measurements of species velocities in an arcjet plume." In Proceedings of the 22nd International Electric Propulsion Conference. IEPC-91-091, October 1991.

${ }^{6}$ J. G. Liebeskind, R. K. Hanson and M. K. Cappelli. "LIF of atomic H in an arcjet thruster." In Proceedings of the 30th AIAA Aerospace Sciences Meeting. AIAA-92-0678, January 1992.

${ }^{7}$ J. G. Liebeskind, R. K. Hanson and M. K. Cappelli. "Flow diagnostics of an arcjet using laser-induced fluorescence." In Proceedings of the 28th Joint Propulsion Conference. AIAA-92-3243, July 1992.

${ }^{8}$ J. G. Liebeskind, R. K. Hanson and M. K. Cappelli. "Laser-induced fluorescence diagnostic for temperature and velocity measurements in a hydrogen arcjet plume." Applied Optics 32(1993) 6117-6127.

${ }^{9}$ D. Keefer et al. "Multiplexed laser induced fluorescence and non-equilibrium processes in arcjets." In Proceedings of the 25th AIAA Plasmadynamics and Lasers Conference. AIAA-94-2656, July 1992.

${ }^{10}$ C. J. Gaeta, R. S. Turley, J. N. Matossian, J. R. Beattie and W. S. Williamson. "Plasma erosion rate diagnostics using laser-induced fluorescence." Review of Scientific Instruments 63(1992) 3090-3095.

${ }^{11}$ C. J. Gaeta, J. N. Matossian, R. S. Turley, J. R. Beattie, J. D. Williams and W. S. Williamson. "Erosion rate diagnostics in ion thrusters using laser-induced fluorescence." Journal of Propulsion and Power 9(1993) 369-376.

${ }^{12}$ D. H. Manzella. "Stationary plasma thruster ion velocity distribution." In Proceedings of the 30th Joint Propulsion Conference. AIAA-94-3141, June 1994.

${ }^{13}$ Hargus, W.A. and M.A. Cappelli, "Interior and exterior laser-induced fluorescence and plasma potential measurements on a laboratory Hall thruster," 35th Joint Propulsion Conference, AIAA-99-2721, June 1999.

${ }^{14}$ E. J. Beiting and J. E. Pollard. "Measurements of xenon ion velocities of the SPT-140 using laser induced fluorescence." In Proceedings of the 3rd International Conference on Spacecraft Propulsion. ESA-SP-465, Oct 2000, 789-797.

${ }^{15}$ W. A. Hargus Jr and M. A. Cappelli. "Laser-induced fluorescence measurements of velocity within a Hall discharge." Applied Physics B B72(2001) 961-969.

${ }^{16} \mathrm{~W}$. A. Hargus Jr. Investigation of the plasma acceleration mechanism within a coaxial Hall thruster. Ph.D. dissertation, Dept. of Mechanical Engineering, Stanford University, March 2001.

${ }^{17}$ N. Dorval, J. Bonnet, J. P. Marque and D. Pigache. "Laser induced fluorescence measurements in xenon plasma thrusters." In Proceedings of the 3rd International Conference on Spacecraft Propulsion. ESA-SP-465, Oct 2000, 797-805. Quoted in. ${ }^{18}$

${ }^{18}$ N. Dorval, J. Bonnet, J. P. Marque, E. Rosencher, S. Chable, F. Rogier and P. Lasgorciex. "Determination of the ionization and acceleration zones in a stationary plasma thruster by optical spectroscopy study: Experiments and model." Journal of Applied Physics 91(2002) 4811-4817.

${ }^{19}$ R. J. Cedolin, W. A. Hargus, P. V. Storm, R. K. Hanson and M. K. Cappelli. "Laser-induced fluorescence study of a xenon Hall thruster." In Proceedings of the 33rd Joint Propulsion Conference. AIAA-97-3053, July 1997.

${ }^{20}$ R. J. Cedolin, W. A. Hargus, P. V. Storm, R. K. Hanson and M. K. Cappelli. "Laser-induced fluorescence study of a xenon Hall thruster." Applied Physics B 65(1997) 459-469.

${ }^{21}$ R. J. Cedolin. Laser-induced fluorescence diagnostics of xenon plasmas. Ph.D. dissertation, Dept. of Mechanical Engineering, Stanford University, June 1997.

${ }^{22}$ D. Keefer. "Multiplexed LIF and Langmuir probe diagnostic measurements in the TAL D-55 thruster." In Proceedings of the 35th Joint Propulsion Conference. AIAA-99-2425, June, 1999.

${ }^{23}$ G. J. Williams Jr., T. B. Smith, F. S. Gulczinski III, B. E. Beal, A. D. Gallimore, and R. P. Drake. "Laser induced fluorescence measurement of ion velocities in the plume of a Hall effect thruster." In Proceedings of the 35th Joint Propulsion Conference. AIAA-99-2424, June 1999.

${ }^{24}$ G. J. Williams Jr., T. B. Smith, M. T. Domonkos, K. J. Shand, A. D. Gallimore and R. P. Drake. "Laser induced fluorescence measurement of ion emitted from hollow cathode." In Proceedings of the 35th Joint Propulsion Conference. AIAA-99-2862, June 1999.

${ }^{25} \mathrm{G}$. J. Williams Jr. "The use of laser-induced fluorescence to characterize discharge cathode erosion in a $30 \mathrm{~cm}$ ring-cusp ion thruster." Ph.D. dissertation, Dept. of Aerospace Engineering, University of Michigan, October 2000.

${ }^{26}$ N. Sadeghi, N. Dorval, J. Bonnet, D. Pigache, C. Kadlec-Phillipe, and A. Bouchoule. "Velocity measurement of Xe ${ }^{+}$in stationary plasma thruster using LIF." In Proceedings of the 35th Joint Propulsion Conference. AIAA-99-2429, June 1999.

${ }^{27}$ Smith, T.B., D.A. Herman, A.D. Gallimore and R.P. Drake, "Deconvolution of axial velocity distributions from Hall thruster LIF spectra," 27th International Electric Propulsion Conference, IEPC-01-0019, October 2001.

${ }^{28}$ T. B. Smith. Deconvolution of ion velocity distributions from laser-induced fluorescence spectra of xenon electrostatic thruster plumes. Ph.D. dissertation, Dept. of Aerospace Engineering, University of Michigan, January 2003.

${ }^{29}$ R. Koslover and R. McWilliams. "Measurement of multidimensional ion velocity distributions by optical tomography." Review of Scientific Instruments 57(1986) 2441-2448.

${ }^{30}$ R. A. Dressler, J. P. M. Beijers, H. Meyer, S. M. Penn, V. M. Bierbaum and S. R. Leone. "Laser probing of ion velocity distributions in drift fields: Parallel and perpendicular temperatures and mobility for $\mathrm{Ba}^{+}$in He." Journal of Chemical Physics 89(1988) 4707-4715.

${ }^{31}$ M. Zintl and R. McWilliams. "Improved optical tomography device." Review of Scientific Instruments 65(1994) 25742579.

${ }^{32}$ D. A. Edrich, R. McWilliams and N. S. Wolf. "Single beam laser induced fluorescence technique for plasma transport measurements." Review of Scientific Instruments 67(1996) 2812-2817.

${ }^{33}$ E. Browne. Table of Isotopes, C. M. Lederer and V. S. Shirley, ed. 7th ed., Wiley, 1978.

${ }^{34}$ C. R. Bingham, M. L. Gaillard, D. J. Pegg, H. K. Carter, R. L. Mlekodaj, J. D. Cole, and P. M. Griffin. "Collinear fast-beam laser spectroscopy experiment: measurement of hyperfine structure and isotope shifts in Xe II." Nuclear Instruments and Methods 202(1982) 147-152. 
${ }^{35}$ G. Borghs, P. De Bisschop, R. E. Silerans, M. Van Hove, and J. M. Van den Cruyce. "Hyperfine structures and isotope shifts of the $5 d^{4} D_{7 / 2}-6 p{ }^{4} P_{5 / 2}^{0}$ transition in xenon ions." Zeitschrift fur Physik A 299(1981) 11-13.

${ }^{36}$ H. Kopfermann. Nuclear Moments. Academic Press, 1958. 2001.

${ }^{37}$ S. Svanberg. Atomic and Molecular Spectroscopy: Basic Aspects and Practical Applications. 3rd ed., Springer-Verlag,

${ }^{38}$ L. Bröstrom, A. Kastberg, J. Lidberg, and S. Mannervik. "Hyperfine-structure measurements in Xe II." Physical Review A 53(1996) 109-112.

${ }^{39}$ C. C. Candler. Atomic Spectra and the Vector Model. Van Nostrand, 1964.

${ }^{40}$ J. T. Verdeyen. Laser Electronics. 3rd ed., Prentice-Hall, 1995.

${ }^{41}$ P. A. Jansson. Deconvolution of images and spectra. Academic Press, 1997.

${ }^{42}$ G. C. Soulas. "Design and performance of $40 \mathrm{~cm}$ ion optics." 27th International Electric Propulsion Conference, IEPC01-090, October 2001. 